\title{
Torus and Subharmonic Motions of a Forced Vibration System in 1:5 Weak Resonance
}

\author{
Yong Guo \\ School of Civil Engineering, Guizhou Institute of Technology, Guiyang, China \\ Correspondence should be addressed to Yong Guo; gy-gates@163.com
}

Received 27 April 2020; Revised 25 July 2020; Accepted 5 August 2020; Published 26 September 2020

Guest Editor: Yu-Hao Sun

Copyright () 2020 Yong Guo. This is an open access article distributed under the Creative Commons Attribution License, which permits unrestricted use, distribution, and reproduction in any medium, provided the original work is properly cited.

\begin{abstract}
The Neimark-Sacker bifurcation of a forced vibration system is considered in this paper. The series solution to the motion equation is obtained, and the Poincaré map is established. The fixed point of the Poincaré map is guaranteed by the implicit function theorem. The map is transformed into its normal form at the fifth-order resonance case. For some parameter values, there exists the torus $T^{1}$. Furthermore, the phenomenon of phase locking on the torus $T^{1}$ is investigated and the parameter condition under which there exists subharmonic motion on the torus $T^{1}$ is determined.
\end{abstract}

\section{Introduction}

In this article, we investigate the torus and subharmonic motions of the following system:

$$
\left\{\begin{array}{l}
\dot{y}_{1}=-y_{2} \\
\dot{y}_{2}=y_{1}+a_{1} \mu y_{2}+b y_{2}^{2}+s y_{1}^{3}+c y_{2}^{3}+\varepsilon f((q+\delta) t)+\text { h.o.t. }
\end{array}\right.
$$

where $\mu, \varepsilon$, and $\delta$ are small parameters; $f$ is a $2 \pi$ periodic function; and $a_{1}, s, b$, and $c$ are constants. $(q+\delta)$ is the frequency of the external force. If $q$ is a positive integer with $q$ $\geq 5$, we say that the system (1) is in $1: q$ weak resonance. For $q$ being a positive integer satisfying $q \leq 4$, the system (1) is referred to as $1: q$ strong resonance. h.o.t. represents the "higher-order terms" than those that have been written out, and the same is true below. There are some mechanical models whose dynamical behaviors can be described by Equation (1), for example, the system shown in Figure 1, see Ref. [1] for more information. In this paper, we investigate abstractly system (1) and give a method for analyzing its Neimark-Sacker bifurcation. The value of relevant param- eters and constants depends on some specific mechanical models whose dynamics can be described by Equation (1). Therefore, we do not introduce the given parameters or constants here, and only choose several sets of values for numerical simulations in Section 4.

For $\varepsilon=0$, Equation (1) undergoes the Hopf bifurcation under certain conditions, and then, for $\varepsilon \neq 0$ and $|\varepsilon|$, sufficiently small torus or the $q$ th order subharmonic motions can occur to Equation (1). The problem of $1: q$ resonance of a closed orbit in $R^{3}$ (or in $C \times S^{1}$ ) leads to the study of the $Z_{q}$-equivariant planar vector (see Refs. $[2,3]$ ) whose versal unfolding has been studied for $q \neq 4$ and been conjectured for $q=4$ by Arnold [2]. Bifurcation sequence inventory at $1: 4$ resonance has been presented by Krauskopf [3]. Gambaudo [4] considered the general study of the periodic perturbation of a one family of autonomous differential equations in the plane satisfying conditions for a generic Hopf bifurcation. Iooss [5] investigated the subharmonic motion in the $1: 3$ and $1: 4$ resonance case. Wan [6] analyzed the Neimark-Sacker bifurcation in the $1: 4$ strong resonance case for the planar map. The width of the resonance tongue at a distance $\sigma$ from the unit circle given by Arnold $[2,7]$ is 


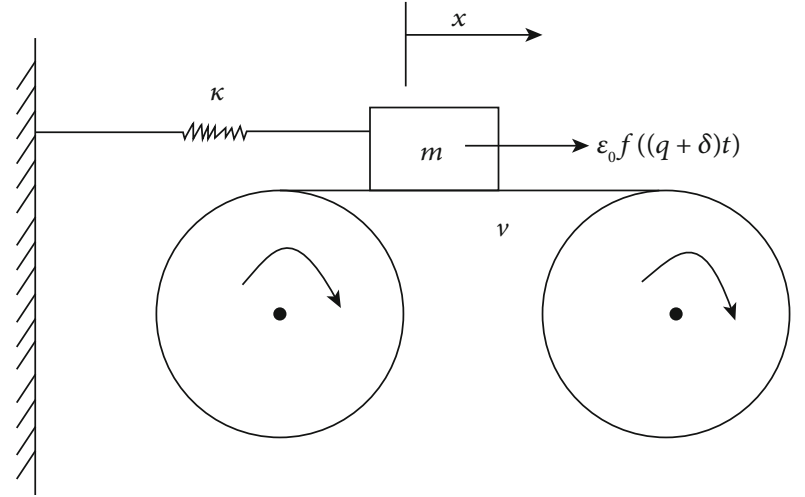

FIgURE 1: Schematic diagram of the dynamic model in Ref. [1].

of the order of $\sigma^{(q-2) / 2}$. Shilnikov et al. [8] and Iooss [9] computed the Arnold tongue in weak resonance case for the planar map. The Neimark-Sacker bifurcation of an oscillator with dry friction was observed in Ref. [1]. Periodic-impact motions and bifurcations of vibroimpact systems near the $1: 4$ strong resonance point are considered in Ref. [10]. Results on other types of forced vibrations can be found in some literatures, see, for example, Refs. [11-16].

Judging from the above statement, we know that there is a lack of efficient criteria depending on the coefficients of the original differential equations, based on which we can talk about the asymptotic behaviors of trajectory. In this paper, we will restrict our attention to the case of $1: 5$ resonance, namely, $q=5$ in Equation (1), and obtain criteria. For higher-order resonance, the procedure is essentially the same as this case but needs tedious computation.

This paper is organized as follows. In Section 2, the Poincaré map is established according to power series solution to Equation (1). The map is further transformed into its normal form. In Section 3, the Neimark-Sacker bifurcation is investigated. When there is a circle bifurcating from the fixed point, the phenomenon of phase locking on the invariant circle is studied and the parameter region in which subharmonic motion can occur is determined. In Section 4, choosing a set of parameters, the theoretical results stated above are verified by numerical simulations.

\section{The Poincaré Map}

Let $z=y_{1}+i y_{2}$, Equation (1) takes the form

$$
\begin{aligned}
\dot{z}= & i z+\frac{a_{1} \mu}{2}(z-\bar{z})-\frac{i b}{4}(z-\bar{z})^{2}+\frac{i s}{8}(z+\bar{z})^{3}-\frac{c}{8}(z-\bar{z})^{3} \\
& +i \varepsilon f((5+\delta) t)+\text { h.o.t. }
\end{aligned}
$$

By the procedure applied in Ref. [1], the solution of Equation (2) satisfying $z(\eta, 0, \mu, \varepsilon)=\eta$ can be written as

$$
z(\eta, t, \mu, \varepsilon)=z_{0}(\eta, t)+\mu z_{10}(\eta, t)+\varepsilon z_{01}(\eta, t)+\text { h.o.t. },
$$

where

$$
\begin{aligned}
z_{0}= & L_{10} \eta+L_{01} \bar{\eta}+L_{20} \eta^{2}+L_{11} \eta \bar{\eta}+L_{02} \bar{\eta}^{2}+L_{30} \eta^{3}+L_{21} \eta^{2} \bar{\eta}+L_{12} \eta \eta^{2}+L_{03} \bar{\eta}^{3}+L_{04} \bar{\eta}^{4}, \\
z_{10}= & \frac{1}{2} e^{i t}\left[\eta t+\frac{i \bar{\eta}}{2}\left(1-e^{-2 i t}\right)\right], \\
z_{01}= & i e^{i t} \int_{0}^{t} e^{-i \tau} f((5+\delta) \tau) d \tau, \\
L_{10}= & e^{i t}, \\
L_{01}= & 0, \\
L_{20}= & \frac{b}{4}\left(-e^{2 i t}+e^{i t}\right), \\
L_{11}= & \frac{b}{2}\left(e^{i t}-1\right), \\
L_{02}= & \frac{b}{12}\left(-e^{i t}+e^{-2 i t}\right), \\
L_{30}= & \left(\frac{b^{2}}{12}+\frac{s}{16}+\frac{i c}{16}\right) e^{3 i t}-\frac{b^{2}}{8} e^{2 i t}-\left(\frac{s}{16}+\frac{i c}{16}\right) e^{i t}+\frac{b^{2}}{24}, \\
L_{21}= & -\frac{b^{2}}{4} e^{2 i t}+\left(\frac{3 c}{8}+\frac{3 s i}{8}-\frac{b^{2}}{6} i\right) t e^{i t}+\frac{23 b^{2}}{36} e^{i t}-\frac{3}{8} b^{2}-\frac{b^{2}}{72} e^{-2 i t}, \\
& +\left(-\frac{b^{3}}{32}-\frac{3 b s}{128}+\frac{3 b c}{128} i\right) e^{-3 i t}+\left(\frac{13 b^{3}}{720}+\frac{7 b s}{320}-\frac{11 b c}{320} i\right) e^{-4 i t} . \\
L_{12}= & \frac{b^{2}}{24} e^{2 i t}+\left(\frac{b^{2}}{6}+\frac{3 s}{16}+\frac{3 c}{16} i\right) e^{i t}-\frac{3 b^{2}}{8}+\left(\frac{b^{2}}{12}-\frac{3 s}{16}-\frac{3 c}{16} i\right) e^{-i t}+\frac{b^{2}}{12} e^{-2 i t}, \\
L_{03}= & -\left(\frac{b^{2}}{24}-\frac{s}{32}+\frac{i c}{32}\right) e^{i t}+\frac{b^{2}}{24}+\frac{b^{2}}{24} e^{-2 i t}-\left(\frac{b^{2}}{24}+\frac{s}{32}-\frac{i c}{32}\right) e^{-3 i t}, \\
L_{04}= & -\frac{b^{3}}{576} e^{2 i t}+\left(-\frac{7}{480} b^{3}+\frac{23}{1920} b s-\frac{11}{480} b c i\right) e^{i t}+\left(\frac{b^{3}}{32}-\frac{b s}{64}+\frac{b c i}{128}\right) \\
& \left.+\frac{b c}{64} i\right) e^{-i t}+\left(\frac{b^{3}}{192}-\frac{b s}{96}+\frac{b c}{96} i\right) e^{-2 i t} \\
&
\end{aligned}
$$

Let $t=2 \pi /(5+\delta)[1]$ in (3), we can get the Poincaré map

$$
\eta \longrightarrow z\left(\eta, \frac{2 \pi}{5+\delta}, \mu, \varepsilon\right)
$$

$$
\begin{aligned}
z\left(\eta, \frac{2 \pi}{5+\delta}, \mu, \varepsilon\right)= & z_{0}\left(\eta, \frac{2 \pi}{5+\delta}\right)+\mu z_{10}\left(\eta, \frac{2 \pi}{5+\delta}\right) \\
& +\varepsilon z_{01}\left(\eta, \frac{2 \pi}{5+\delta}\right)+\text { h.o.t. } \\
= & \lambda_{0}\left(1-\frac{2 \pi \delta}{25} i\right) \eta+\frac{\mu a_{1}}{2} \lambda_{0}\left[\frac{2 \pi}{5} \eta+\frac{i \bar{\eta}}{2}\left(1-\bar{\lambda}_{0}^{2}\right)\right] \\
& +\varepsilon i \lambda_{0} \int_{0}^{2 \pi / 5} e^{-i \tau} f((5+\delta) \tau) d \tau+\frac{g_{20}}{2} \eta^{2} \\
& +g_{11} \eta \bar{\eta}+\frac{g_{02}}{2} \bar{\eta}^{2}+\frac{g_{30}}{6} \eta^{3}+\frac{g_{21}}{2} \eta^{2} \bar{\eta}+\frac{g_{12}}{2} \eta \bar{\eta}^{2} \\
& +\frac{g_{03}}{6} \bar{\eta}^{3}+\frac{g_{04}}{24} \bar{\eta}^{4}+\text { h.o.t., }
\end{aligned}
$$


where $\lambda_{0}=e^{(2 \pi / 5) i}, g_{20} / 2, g_{11}, g_{02} / 2, g_{30} / 6, g_{21} / 2, g_{12} / 2, g_{03} / 6$, and $g_{04} / 24$ are given by $L_{20}, L_{11}, L_{02}, L_{30}, L_{21}, L_{12}, L_{03}$, and $L_{04}$, all of which are evaluated at $t=2 \pi /(5+\delta)$.

Because $z(0,2 \pi / 5,0,0)=0$, and

$$
\left(\left.D\left[z\left(\eta, \frac{2 \pi}{5+\delta}, \mu, \varepsilon\right)-\eta\right]\right|_{\eta=0, \delta=0, \mu=0, \varepsilon=0}\right) \varsigma=\left(\lambda_{0}-1\right) \varsigma
$$

$\lambda_{0}-1 \neq 0$, by the implicit function theorem, we know that (6) has a fixed point $F(\delta, \mu, \varepsilon)$ which depends on $\delta, \mu$, and $\varepsilon$ continuously.

$F(\delta, \mu, \varepsilon)=F_{1}+$ h.o.t. where $F_{1}=\left(\varepsilon i \lambda_{0} /\left(1-\lambda_{0}\right)\right) \int_{0}^{2 \pi / 5}$ $e^{-i \tau} f((5+\delta) \tau) d \tau$.

Making a translation of coordinate $\xi=\eta-F(\delta, \mu, \varepsilon)$, map (6) becomes

$$
\begin{aligned}
\xi^{\prime}= & \lambda_{0}\left(1+\frac{\mu a_{1} \pi}{5}+\frac{g_{20} F_{1}+g_{11} \bar{F}_{1}}{\lambda_{0}}-\frac{2 \pi \delta}{25} i\right) \xi+c_{1} \bar{\xi} \\
& +\frac{g_{20}}{2} \xi^{2}+g_{11} \xi \bar{\xi}+\frac{g_{02}}{2} \bar{\xi}^{2}+\frac{g_{30}}{6} \xi^{3}+\frac{g_{21}}{2} \xi^{2} \bar{\xi}+\frac{g_{12}}{2} \xi \bar{\xi}^{2} \\
& +\frac{g_{03}}{6} \bar{\xi}^{3}+\frac{g_{04}}{24} \bar{\xi}^{4}+\text { h.o.t. }
\end{aligned}
$$

With a change of coordinate $\xi=(1+O(\delta, \mu, \varepsilon)) \zeta$, we can eliminate the linear term of $\bar{\xi}$ and not change the others in map (8). It follows that

$$
\begin{aligned}
\xi^{\prime}= & \lambda_{0}\left(1+\frac{\mu a_{1} \pi}{5}+\frac{g_{20} F_{1}+g_{11} \bar{F}_{1}}{\lambda_{0}}-\frac{2 \pi \delta}{25} i\right) \xi+\frac{g_{20}}{2} \xi^{2} \\
& +g_{11} \xi \bar{\xi}+\frac{g_{02}}{2} \bar{\xi}^{2}+\frac{g_{30}}{6} \xi^{3}+\frac{g_{21}}{2} \xi^{2} \bar{\xi}+\frac{g_{12}}{2} \bar{\xi}^{2}+\frac{g_{03}}{6} \bar{\xi}^{3} \\
& +\frac{g_{04}}{24} \bar{\xi}^{4}+\text { h.o.t. }
\end{aligned}
$$

By the theory of a normal form for a map, we can cancel all of the nonresonant terms, and map (9) is equivalent to

$$
\begin{aligned}
\omega^{\prime}= & \lambda_{0}\left(1+\frac{\mu a_{1} \pi}{5}+\frac{g_{20} F_{1}+g_{11} \bar{F}_{1}}{\lambda_{0}}-\frac{2 \pi \delta}{25} i\right) \omega+\frac{g_{21}^{\prime}}{2} \omega^{2} \bar{\omega} \\
& +\frac{g_{04}^{\prime}}{24} \bar{\omega}^{4}+\text { h.o.t. }
\end{aligned}
$$

The coefficients of $g_{21}^{\prime} / 2$ and $g_{04}^{\prime} / 24$ are determined by Iooss [5]

$$
\begin{aligned}
& \frac{g_{21}^{\prime}}{2}=\frac{g_{21}}{2}-g_{20} \gamma_{11}-g_{11}\left(\bar{\gamma}_{11}+\frac{\gamma_{20}}{2}\right)-g_{02} \frac{\bar{\gamma}_{02}}{2} \\
& +\lambda \gamma_{20}\left(g_{11}-\lambda \gamma_{11}\right)+\gamma_{11} \bar{\lambda}\left(\frac{g_{20}}{2}-\frac{\lambda \gamma_{20}}{2}\right) \\
& +\gamma_{11} \lambda\left(\bar{g}_{11}-\bar{\lambda} \bar{\gamma}_{11}\right)+\gamma_{02} \bar{\lambda}\left(\frac{\bar{g}_{02}}{2}-\frac{\bar{\lambda} \bar{\gamma}_{02}}{2}\right) \\
& +\lambda\left[\gamma_{20} \gamma_{11}+\gamma_{11}\left(\frac{\gamma_{20}}{2}+\bar{\gamma}_{11}\right)+\frac{\gamma_{02} \bar{\gamma}_{02}}{2}\right], \frac{g_{04}^{\prime}}{24} \\
& =-\lambda \gamma_{11}\left(\frac{\gamma_{11} \gamma_{02}}{2}+\frac{\gamma_{02} \bar{\gamma}_{20}}{2}\right)-\lambda \gamma_{02}\left(\frac{\bar{\gamma}_{20}^{2}}{2}+\frac{\bar{\gamma}_{11} \gamma_{02}}{2}\right) \\
& -\frac{\lambda \gamma_{20}}{2} \frac{\gamma_{02}^{2}}{4}-\lambda \gamma_{11} \frac{\gamma_{02} \bar{\gamma}_{20}}{4}-\frac{\lambda \gamma_{02}}{2} \frac{\bar{\gamma}_{20}^{2}}{4}+\frac{g_{20}}{2} \frac{\gamma_{02}^{2}}{4} \\
& +g_{11} \frac{\gamma_{02}}{2} \frac{\bar{\gamma}_{20}}{2}+\frac{g_{02}}{2} \frac{\bar{\gamma}_{20}^{2}}{4}+g_{11}\left(\frac{\gamma_{11} \gamma_{02}}{2}+\frac{\gamma_{02} \bar{\gamma}_{20}}{2}\right) \\
& +g_{02}\left(\frac{\bar{\gamma}_{20}^{2}}{2}+\frac{\bar{\gamma}_{11} \gamma_{02}}{2}\right)-\frac{g_{12}}{2} \frac{\gamma_{02}}{2}-\frac{g_{03}}{2} \frac{\bar{\gamma}_{20}}{2} \\
& +\frac{\gamma_{20}}{2}\left(\frac{g_{02}}{2}-\frac{\lambda \gamma_{02}}{2}\right)^{2}+\gamma_{11}\left[\lambda \bar{\lambda}\left(\frac{\gamma_{11} \gamma_{02}}{2}+\frac{\gamma_{02} \bar{\gamma}_{20}}{2}\right)\right. \\
& -\bar{\lambda} g_{11} \frac{\gamma_{02}}{2}-\bar{\lambda} g_{02} \frac{\bar{\gamma}_{20}}{2}+\bar{\lambda} \frac{g_{03}}{6}+\frac{g_{02} \bar{g}_{20}}{4}-\bar{\lambda} \frac{g_{02}}{2} \frac{\bar{\gamma}_{20}}{2} \\
& \left.-\lambda \frac{\bar{g}_{20}}{2} \frac{\gamma_{02}}{2}+\lambda \bar{\lambda} \frac{\gamma_{02}}{2} \frac{\bar{\gamma}_{20}}{2}\right]+\frac{\gamma_{02}}{2}\left[2 \bar{\lambda}^{2}\left(\frac{\bar{\gamma}_{20}^{2}}{2}+\frac{\bar{\gamma}_{11} \gamma_{02}}{2}\right)\right. \\
& -2 \bar{\lambda} \bar{g}_{20} \frac{\bar{\gamma}_{20}}{2}-2 \bar{\lambda} \bar{g}_{11} \frac{\gamma_{02}}{2}+\frac{\bar{\lambda} \bar{g}_{30}}{3}+\left(\frac{\bar{g}_{20}}{2}\right)^{2} \\
& \left.+\bar{\lambda}^{2}\left(\frac{\bar{\gamma}_{20}}{2}\right)^{2}-\bar{\lambda} \bar{g}_{20} \frac{\bar{\gamma}_{20}}{2}\right]+\frac{g_{04}}{24},
\end{aligned}
$$

where $\lambda=\lambda_{0}\left(1+\left(\mu a_{1} \pi / 5\right)+\left(\left(g_{20} F_{1}+g_{11} \bar{F}_{1}\right) / \lambda_{0}\right)-(2 \pi \delta /\right.$ 25)i), and

$$
\begin{aligned}
& \gamma_{20}=\frac{g_{20}}{\lambda-\lambda^{2}}, \\
& \gamma_{11}=\frac{g_{11}}{\lambda-\lambda \bar{\lambda}}, \\
& \gamma_{02}=\frac{g_{02}}{\lambda-\bar{\lambda}^{2}} .
\end{aligned}
$$

After computing straightly, we have

$$
\begin{gathered}
\frac{g_{21}^{\prime}(0)}{2}=\left(\frac{3 c}{8}+\frac{3 s i}{8}-\frac{b^{2} i}{6}\right) \frac{2 \pi}{5} \lambda_{0} \\
\frac{g_{04}^{\prime}(0)}{24}=-\frac{b c}{128} i+\frac{b c}{128} i \lambda_{0} .
\end{gathered}
$$




\section{Invariant Circle and Phase Locking}

For convenience, let us write (10) as

$$
\omega^{\prime}=\lambda \omega+\alpha \omega^{2} \bar{\omega}+\gamma \bar{\omega}^{4}+\text { h.o.t. }
$$

where $\alpha=g_{21}^{\prime} / 2$ and $\gamma=g_{04}^{\prime} / 24$. Let $\lambda=\lambda_{0}\left(1+\tilde{\lambda}_{1}\right), \alpha_{0}=$ $\left.\alpha\right|_{\mu=\varepsilon=\delta=0}$, and $\gamma_{0}=\left.\gamma\right|_{\mu=\varepsilon=\delta=0}$.

As we know, the dynamic behavior of map (14) is corresponding to that of Equation (1). If $\operatorname{Re}\left(\alpha_{0} \bar{\lambda}_{0}\right) \neq 0$ and the derivation of $\operatorname{Re}\left(\tilde{\lambda}_{1}\right)$ with respect to parameters is nonzero, the Neimark-Sacker bifurcation takes place for map (14).

We further study the phenomenon of phase locking on an invariant circle bifurcating from the fixed point of map (14). By changing variables $\omega=r e^{i \varphi}$ and $\omega^{\prime}=R e^{i \Phi}$, map (14) is described by Shilnikov et al. [8]

$$
R=\left[1+\operatorname{Re}\left(\tilde{\lambda}_{1}\right)\right] r+\operatorname{Re}\left(\alpha_{0} \bar{\lambda}_{0}\right) r^{3}+\operatorname{Re}\left(\gamma_{0} \bar{\lambda}_{0} e^{-5 i \varphi}\right) r^{4}+\text { h.o.t. }
$$

$$
\Phi=\varphi+\theta+\operatorname{Im}\left(\alpha_{0} \bar{\lambda}_{0}\right) r^{2}+\operatorname{Im}\left(\gamma_{0} \bar{\lambda}_{0} e^{-5 i \varphi}\right) r^{3}+\text { h.o.t. }
$$

where $\theta=\arg (\lambda)$.

Let us note $l_{1}=\operatorname{Re}\left(\alpha_{0} \bar{\lambda}_{0}\right), m_{1}=\operatorname{Im}\left(\alpha_{0} \bar{\lambda}_{0}\right), A=\operatorname{Re}\left(\gamma_{0} \bar{\lambda}_{0}\right)$, $B=\operatorname{Im}\left(\gamma_{0} \bar{\lambda}_{0}\right)$, and $\psi=\arctan (A / B)$. Equations (15) and (16) can be written as

$$
R=\left[1+\operatorname{Re}\left(\tilde{\lambda}_{1}\right)\right] r+l_{1} r^{3}+\sqrt{A^{2}+B^{2}} \cos (5 \varphi+\psi) r^{4}+\text { h.o.t. }
$$

$$
\Phi=\varphi+\theta+m_{1} r^{2}-\sqrt{A^{2}+B^{2}} \sin (5 \varphi+\psi) r^{3}+\text { h.o.t. }
$$

In order to find the fifth-order subharmonic solution, iterating Equations (17) and (18) five times, then we obtain

$$
\begin{aligned}
& R=\left[1+5 \operatorname{Re}\left(\tilde{\lambda}_{1}\right)\right] r+5 l_{1} r^{3}+5 \sqrt{A^{2}+B^{2}} \cos (5 \varphi+\psi) r^{4}+\text { h.o.t. } \\
& \Phi=\varphi+5 \theta+5 m_{1} r^{2}-5 \sqrt{A^{2}+B^{2}} \sin (5 \varphi+\psi) r^{3}+\text { h.o.t. }
\end{aligned}
$$

Solutions of equations

$$
\left\{\begin{array}{l}
R=r \\
\Phi=\varphi(\bmod 2 \pi)
\end{array}\right.
$$

are corresponding to the period 5 points of map (14).

Solving the first equation of (20), we get

$$
R=\sqrt{\frac{\operatorname{Re}\left(\tilde{\lambda}_{1}\right)}{\left|l_{1}\right|}}+\frac{\sqrt{A^{2}+B^{2}} \operatorname{Re}\left(\tilde{\lambda}_{1}\right)}{2 l_{1}^{2}} \cos (5 \varphi+\psi)+\text { h.o.t. }
$$

Substituting the second equation of (20) with (21), and considering that $\theta$ is close to $2 \pi / 5$, we have

$$
\begin{aligned}
& \theta-\frac{2 \pi}{5}+m_{1} \frac{\operatorname{Re}\left(\tilde{\lambda}_{1}\right)}{\left|l_{1}\right|}+\left[\frac{m_{1}}{\left|l_{1}\right|} \cos (5 \varphi+\psi)\right. \\
& -\sin (5 \varphi+\psi)]\left[\frac{\operatorname{Re}\left(\tilde{\lambda}_{1}\right)}{\left|l_{1}\right|}\right]^{3 / 2} \sqrt{A^{2}+B^{2}}+\text { h.o.t. }=0 .
\end{aligned}
$$

If for some $\varphi=\varphi_{0}$,

$$
\begin{aligned}
& \theta-\frac{2 \pi}{5}+\frac{\operatorname{Re}\left(\tilde{\lambda}_{1}\right) m_{1}}{\left|l_{1}\right|} \\
& +\frac{\sqrt{m_{1}^{2}+l_{1}^{2}} \sqrt{A^{2}+B^{2}}\left[\operatorname{Re}\left(\tilde{\lambda}_{1}\right)\right]^{3 / 2}}{\left|l_{1}\right|^{5 / 2}} \sin \left(5 \varphi_{0}+\psi_{1}\right)=0
\end{aligned}
$$

together with $\sqrt{A^{2}+B^{2}} \neq 0$, "h.o.t." in Equation (22) can be offset by adjusting $\varphi$ near $\varphi_{0}$.

For the boundedness of sinusoidal function and $\theta=(2 \pi$ /5) $-(2 \pi \delta / 25)$, we assert that if

$$
\left|\frac{\operatorname{Re}\left(\tilde{\lambda}_{1}\right) m_{1}}{\left|l_{1}\right|}-\frac{2 \pi \delta}{25}\right|<\frac{\sqrt{m_{1}^{2}+l_{1}^{2}} \sqrt{A^{2}+B^{2}}\left[\operatorname{Re}\left(\tilde{\lambda}_{1}\right)\right]^{3 / 2}}{\left|l_{1}\right|^{5 / 2}}
$$

there exist two sets of values of $\varphi$ denoted by $\left(\varphi_{1}^{1}, \varphi_{2}^{1}, \varphi_{3}^{1}, \varphi_{4}^{1}\right.$ ,$\left.\varphi_{5}^{1}\right)$ and $\left(\varphi_{1}^{2}, \varphi_{2}^{2}, \varphi_{3}^{2}, \varphi_{4}^{2}, \varphi_{5}^{2}\right)$ which satisfy Equation (22). Then, the second equation of (20) follows and map (14) possesses two sets of period 5 solutions, one of which is stable, another is unstable.

\section{The Numerical Simulations}

To illustrate the results stated above, numerical simulations will be presented in this section. As mentioned in the introduction, $f$ is a $2 \pi$ periodic function. For simplicity, let $f((5+\delta) t)=\sin ((5+\delta) t)$ in Equation (1), which is a simple form of $f((5+\delta) t)$. It follows from (6)-(14) that

$$
\begin{aligned}
\omega^{\prime}= & \lambda_{0}\left(1+\frac{\mu a_{1} \pi}{5}-\frac{5 b \varepsilon}{24} \sin \frac{2 \pi}{5}-\frac{2 \pi \delta}{25} i\right) \omega+\alpha \omega^{2} \bar{\omega} \\
& +\gamma \bar{\omega}^{4}+\text { h.o.t. }
\end{aligned}
$$

Furthermore, we take the set of parameters $a_{1}=2, b=1$, $s=0, c=-4$. We can calculate that $\operatorname{Re}\left(\alpha_{0} \bar{\lambda}_{0}\right)=-1.8850<0$ and the derivation of $\operatorname{Re}\left(\tilde{\lambda}_{1}\right)$ with respect to parameters $\mu$ and $\varepsilon$ is nonzero, which means that the supercritical Neimark-Sacker bifurcation takes place for map (25) [17]. 


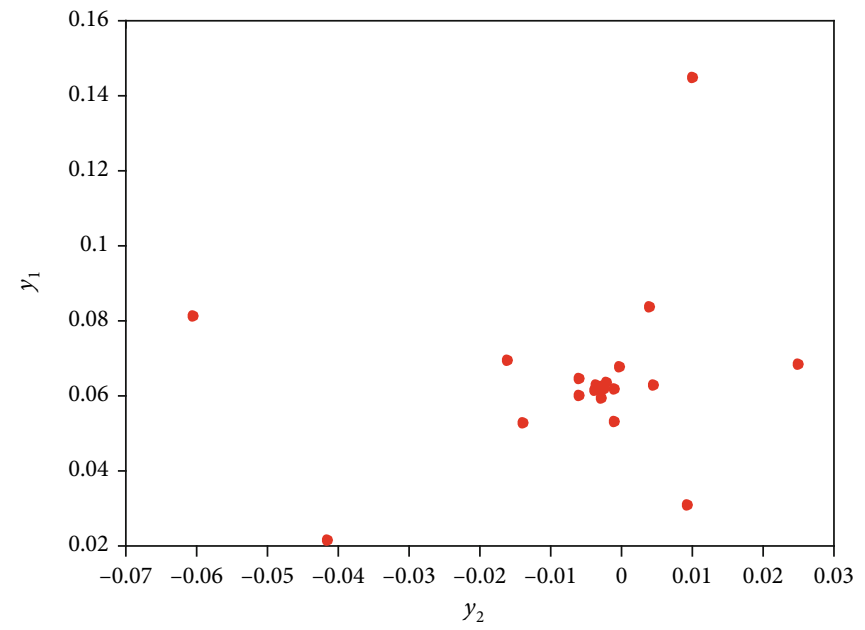

(a)

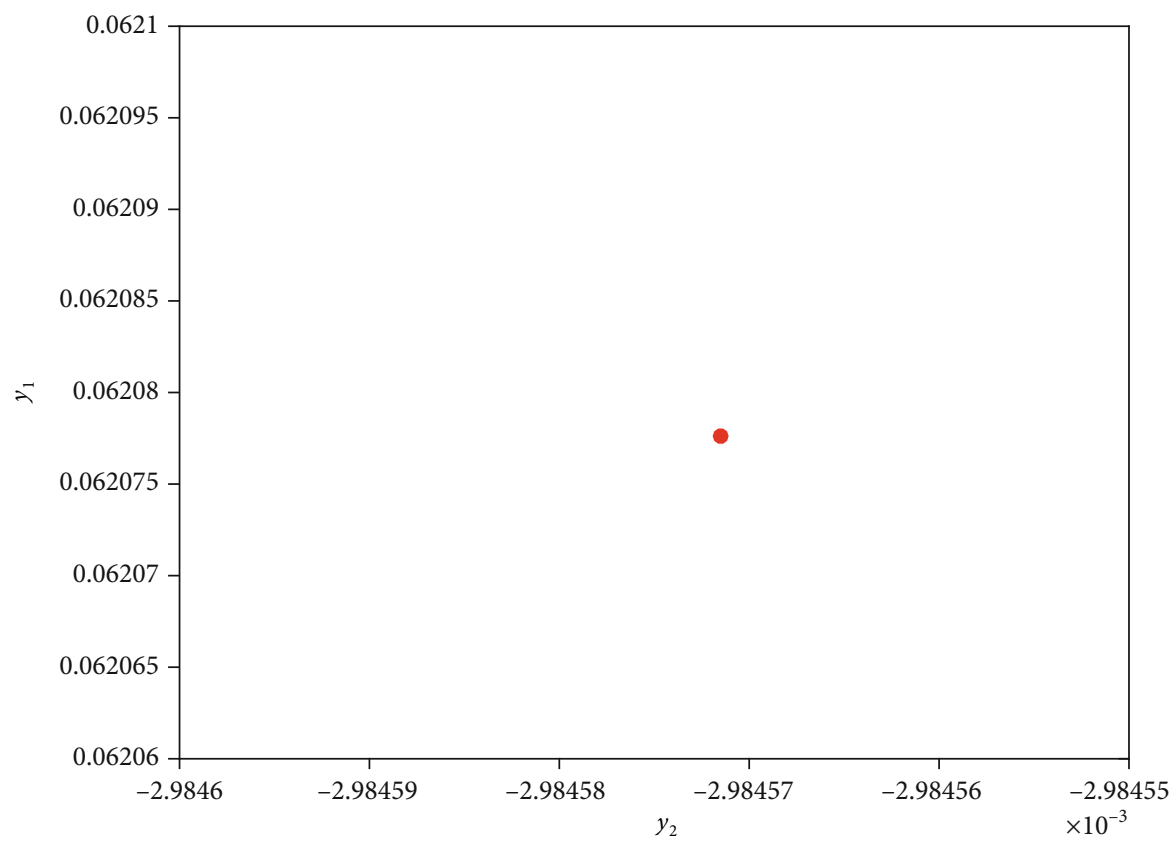

(b)

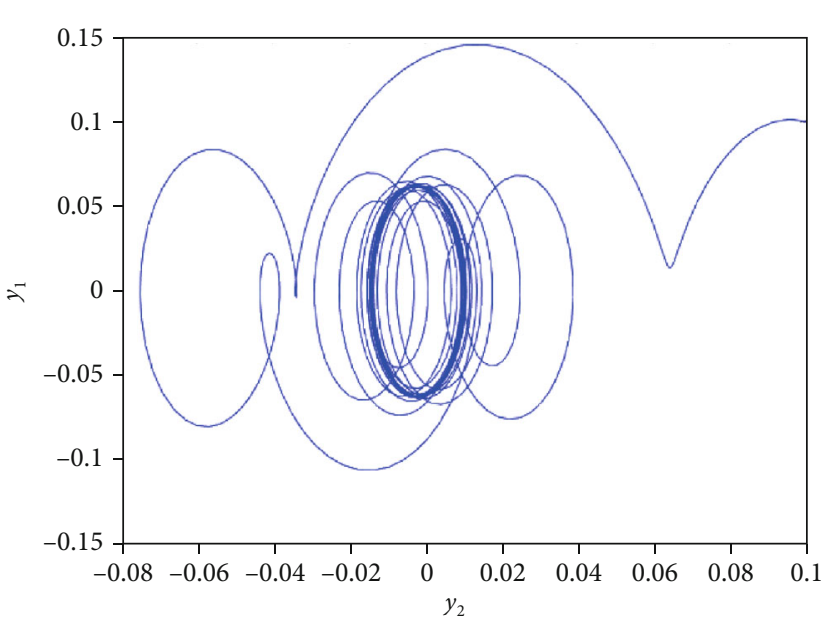

(c)

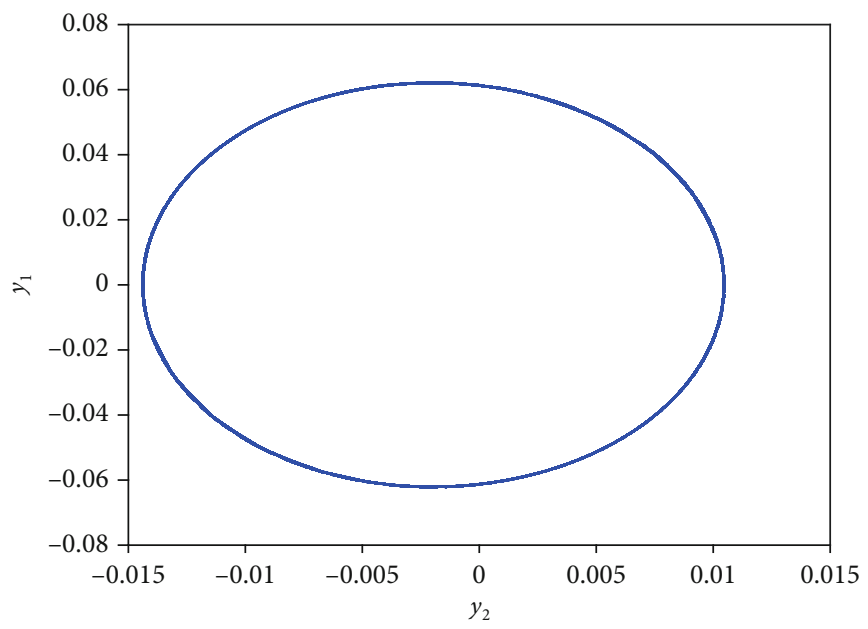

(d)

Figure 2: (a) Poincaré map; (b) Poincaré map (last 5000 points); (c) phase diagram; (d) phase diagram (last 10000 points). 


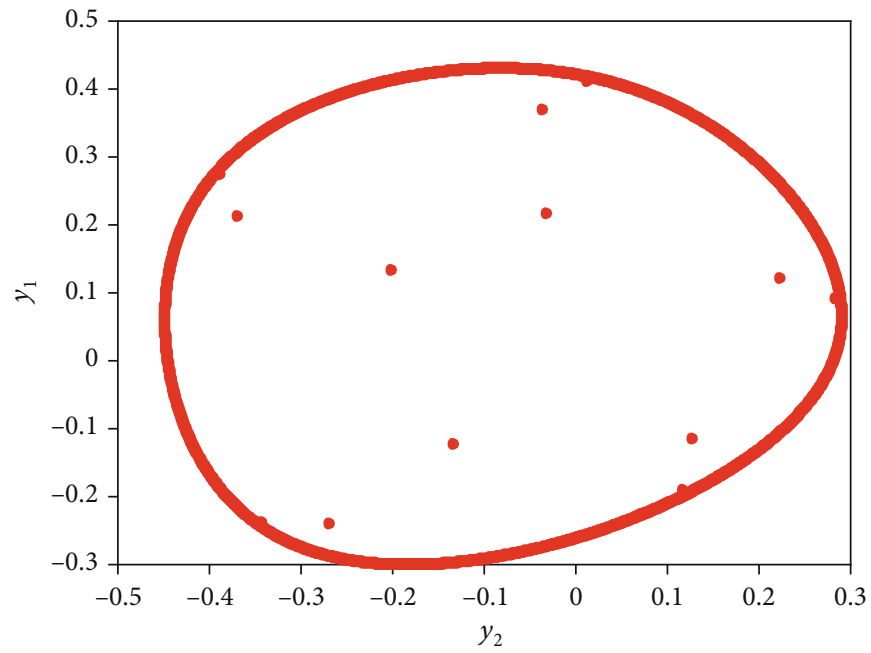

(a)

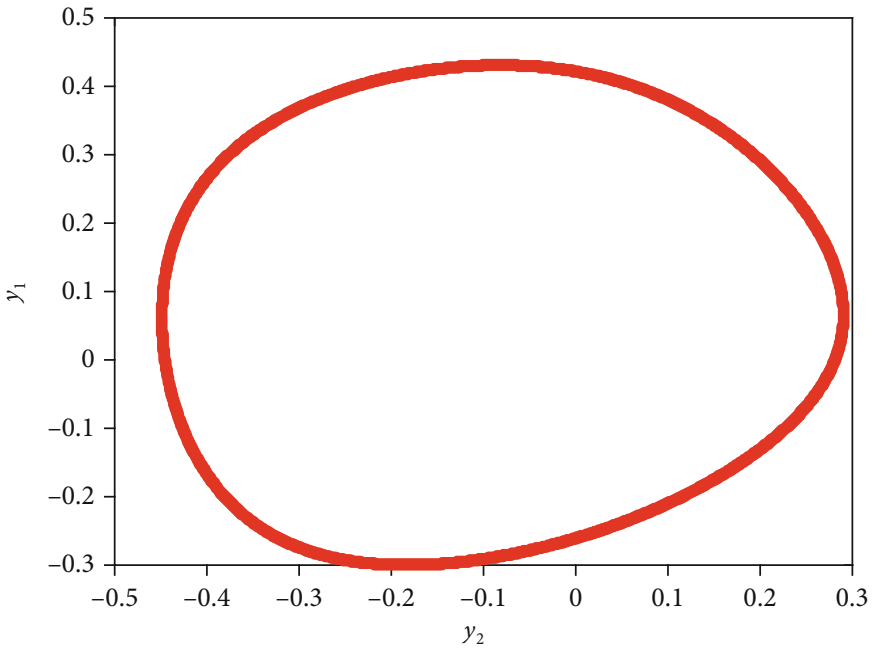

(b)

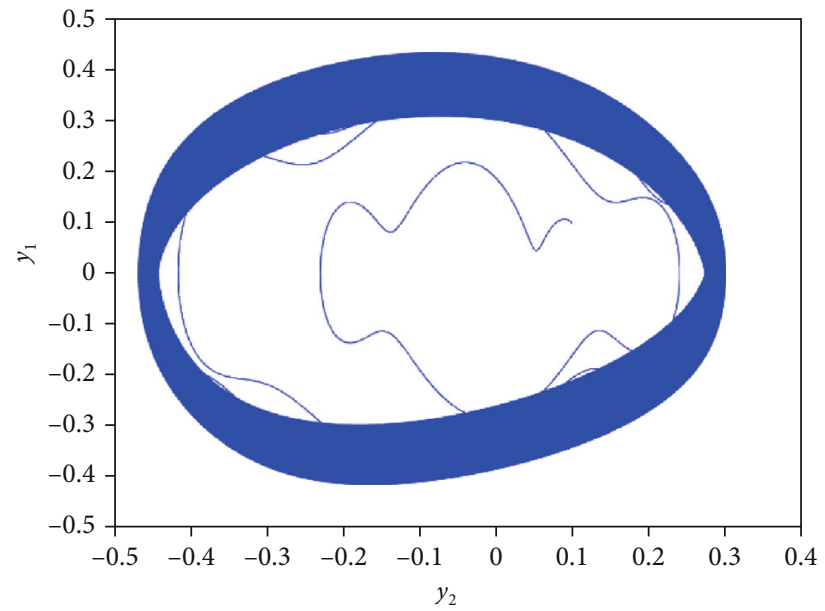

(c)

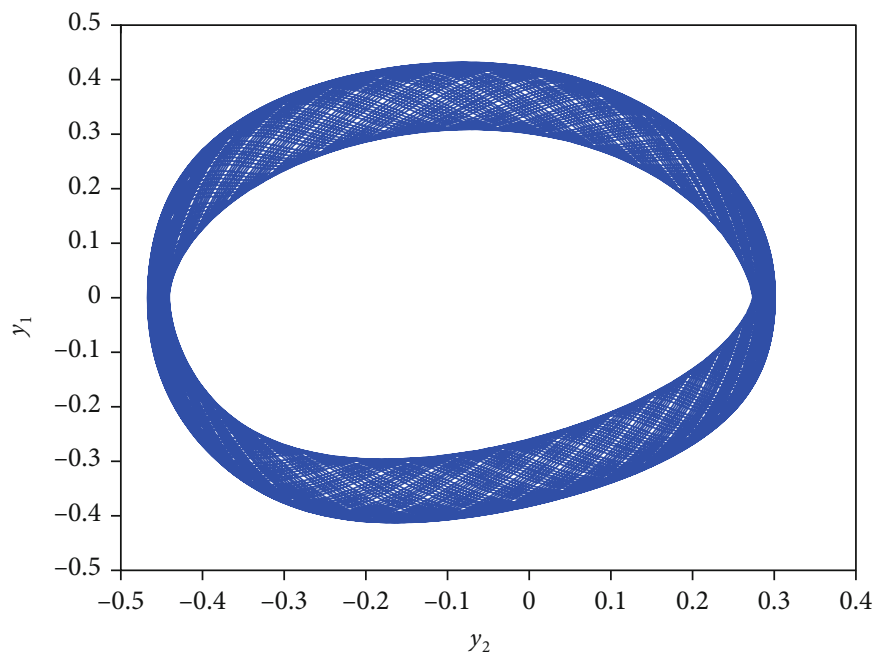

(d)

Figure 3: (a) Poincaré map; (b) Poincaré map (last 5000 points); (c) phase diagram; (d) phase diagram (last 10000 points). 


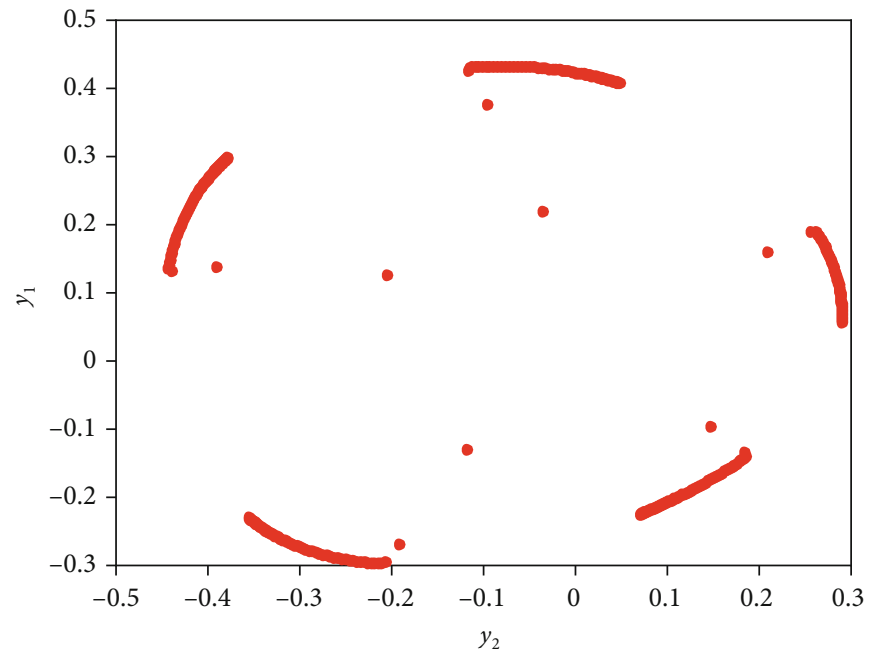

(a)

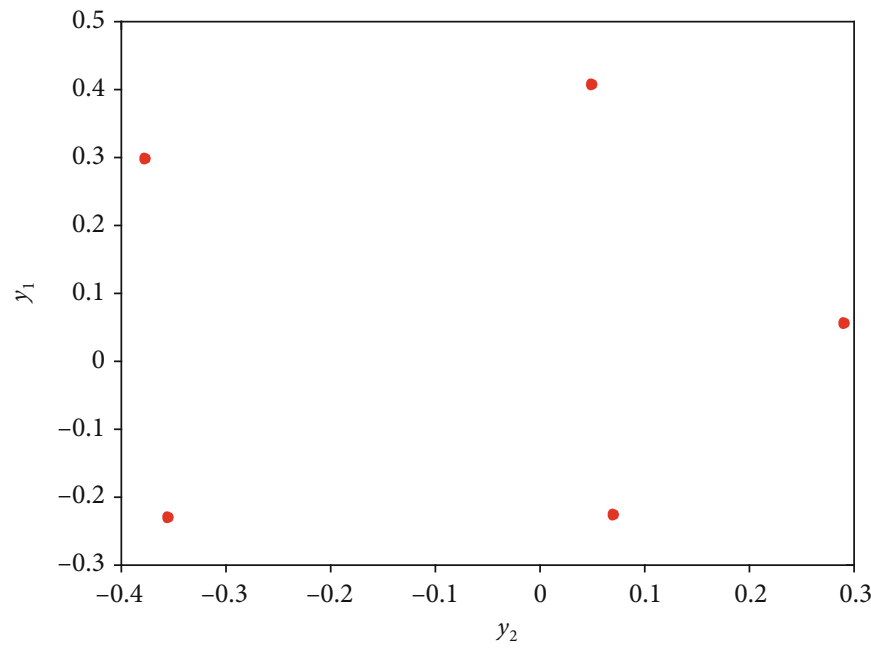

(b)

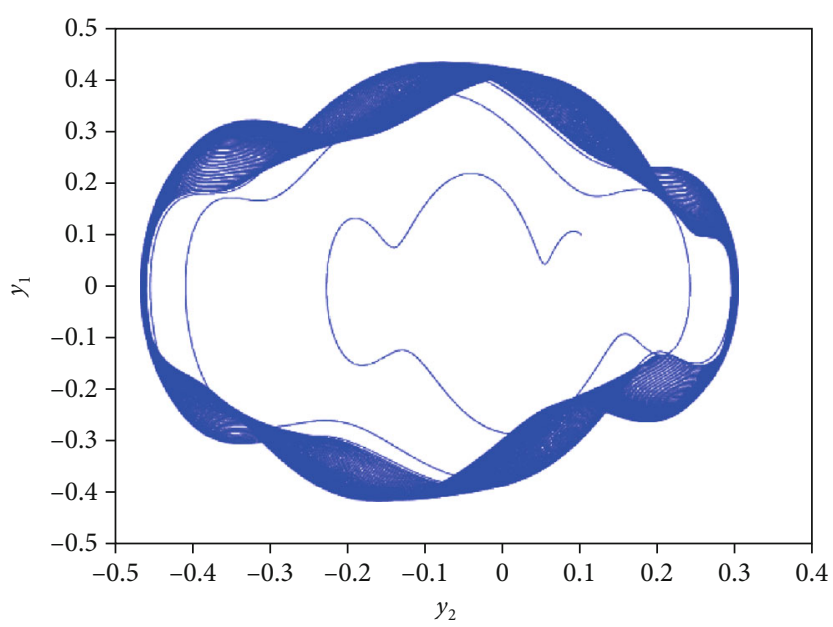

(c)

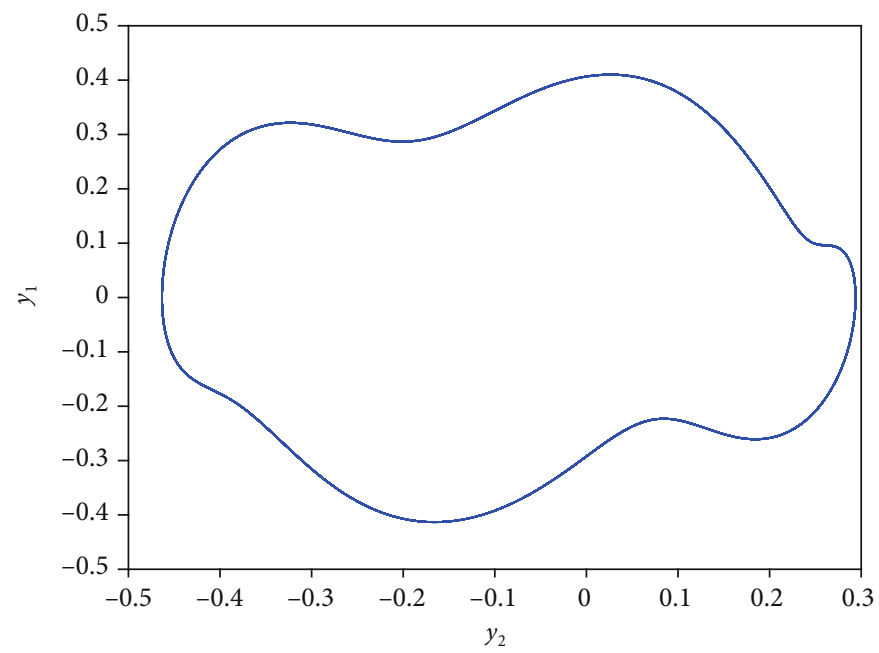

(d)

Figure 4: (a) Poincaré map; (b) Poincaré map (last 5000 points); (c) phase diagram; (d) phase diagram (last 10000 points). 
Choosing $\varepsilon=-0.3, \mu=-0.2$, and $\delta=0$, we get $\operatorname{Re}\left(\tilde{\lambda}_{1}\right)$ $=-0.1919$ and then assert that map (25) possesses a stable fixed point (see Figures 2(a) and 2(b)), namely, a stable periodic solution of Equation (1) (shown in Figures 2(c) and $2(d))$. If the dynamic behaviors of the model in Figure 1 can be described by Equation (1) with the present parameters, the period of vibration of the mass body is the same as the external force.

Choosing $\varepsilon=-0.3$ and $\mu=0.2$, we have $\operatorname{Re}\left(\tilde{\lambda}_{1}\right)=0.3108$ and then assert that map (25) possesses a stable invariant circle [17], namely, a stable torus motion of Equation (1). Because the limitation of map (25) on the stable invariant circle is a circle diffeomorphism, the trajectory on the torus is quasiperiodic or subharmonic motion, which depends on expression (24). As will be investigated below.

For $\delta=0$, By straight computation, we obtain

$$
\begin{gathered}
\frac{\operatorname{Re}\left(\tilde{\lambda}_{1}\right) m_{1}}{\left|l_{1}\right|}-\frac{2 \pi \delta}{25}=-0.0345 \\
\frac{\sqrt{m_{1}^{2}+l_{1}^{2}} \sqrt{A^{2}+B^{2}}\left[\operatorname{Re}\left(\tilde{\lambda}_{1}\right)\right]^{3 / 2}}{\left|l_{1}\right|^{5 / 2}}=0.0025
\end{gathered}
$$

which invalidate (24), there is no period 5 points for map (25) (see Figures 3(a) and 3(b)), and the solution of Equation (1) is quasiperiodic (shown in Figures 3(c) and 3(d)). If the dynamic behaviors of the model in Figure 1 can be described by Equation (1) with the present parameters, the vibration of the mass body is quasiperiodic, which is not periodic motion even though the external force is periodic.

For $\delta=-0.13$, calculation shows

$$
\begin{gathered}
\frac{\operatorname{Re}\left(\tilde{\lambda}_{1}\right) m_{1}}{\left|l_{1}\right|}-\frac{2 \pi \delta}{25}=-0.0019 \\
\frac{\sqrt{m_{1}^{2}+l_{1}^{2}} \sqrt{A^{2}+B^{2}}\left[\operatorname{Re}\left(\tilde{\lambda}_{1}\right)\right]^{3 / 2}}{\left|l_{1}\right|^{5 / 2}}=0.0025 .
\end{gathered}
$$

Expression (24) follows, map (25) possesses period 5 points (see Figures 4(a) and 4(b)), and Equation (1) yields a subharmonic motion (shown in Figures $4(\mathrm{c})$ and $4(\mathrm{~d})$ ). If the dynamic behaviors of the model in Figure 1 can be described by Equation (1) with the present parameters, the vibration of the mass body is subharmonic, whose period is five times than that of the external force.

Because the analysis method of this paper is for system (1) with abstract coefficients, it can be applied in other mechanical models whose dynamics can be described by Equation (1), for example, the forced Van der Pol equations [6], the forced dry friction system [18], the vibration of railway bow net, and the forced vibration of cantilevered flowconveying pipe.

\section{Conclusions}

In this paper, we study the Neimark-Sacker bifurcation of a forced vibration system by theoretical analysis and numerical simulations in the 1:5 resonance case. The Poincaré map is established by the analytical method. By means of analyzing the map, it is shown that there exist quasiperiodic and subharmonic solutions on the torus. Numerical simulations agreed with the theoretical results. It is certain that the method applied in this paper can be applied to some other analogous systems.

\section{Data Availability}

The data used to support the findings of this study are available from the corresponding author upon request.

\section{Conflicts of Interest}

The author declares that they have no conflicts of interest.

\section{Acknowledgments}

This work is supported by XJGC20190909.

\section{References}

[1] Y. Guo and J. H. Xie, "Neimark-Sacker (N-S) bifurcation of oscillator with dry friction in 1:4 strong resonance," Applied Mathematics and Mechanics, vol. 34, no. 1, pp. 27-36, 2013.

[2] V. I. Arnold, "Loss of stability of self-oscillations close to resonance and versal deformations of equivariant vector fields," Functional Analysis and its Applications, vol. 22, pp. 85-92, 1977.

[3] B. Krauskopf, "Bifurcation sequences at 1:4 resonance: an inventory," Nonlinearity, vol. 7, no. 3, pp. 1073-1091, 1994.

[4] J. M. Gambaudo, "Perturbation of a Hopf bifurcation by an external time-periodic forcing," Journal of Differential Equations, vol. 57, no. 2, pp. 172-199, 1985.

[5] G. Iooss, Bifurcation of Maps and Applications, North-Holland Publishing Company, Amsterdam. New York. Oxford, 1979.

[6] Y.-H. Wan, "Bifurcation into invariant tori at points of resonance," Archive for Rational Mechanics Analysis, vol. 68, no. 4, pp. 343-357, 1978.

[7] V. I. Arnold, Geometrical Methods in the Theory of Ordinary Differential Equations, Springe-Verlag, New York, 1983.

[8] L. P. Shilnikov, A. L. Shilnikov, D. V. Turaev, and L. O. Chua, Methods of Qualitative Theory in Nonlinear Dynamics, Part II, World Scientific, 2001.

[9] G. Iooss, Bifurcation and transition to turbulence in hydrodynamics, Springer, Berlin Heidelberg, Berlin, Heidelberg, 1984.

[10] G. Luo, Y. Zhang, J. Xie, and J. Zhang, "Periodic-impact motions and bifurcations of vibro-impact systems near 1:4 strong resonance point," Communications in Nonlinear Science and Numerical Simulation, vol. 13, no. 5, pp. 10021014, 2008.

[11] Y. Guo and A. C. J. Luo, "Periodic motions in a double-well Duffing oscillator under periodic excitation through discrete implicit mappings," International Journal of Dynamics and Control, vol. 5, pp. 223-238, 2015. 
[12] T. S. Amer, M. A. Bek, and I. S. Hamada, "On the motion of harmonically excited spring pendulum in elliptic path near resonances," Advances in Mathematical Physics, vol. 2016, Article ID 8734360, 15 pages, 2016.

[13] Z. Hao, Q. Cao, and M. Wiercigroch, "Nonlinear dynamics of the quasi-zero-stiffness SD oscillator based upon the local and global bifurcation analyses," Nonlinear Dynamics, vol. 87, pp. 987-1014, 2016.

[14] A. C. J. Luo, "Periodic motions and chaos in nonlinear dynamical systems," The European Physical Journal Special Topics, vol. 228, no. 9, pp. 1745-1746, 2019.

[15] N. Hua and T. Li-Xin, "The existence of $\mathrm{n}$ periodic solutions on one element n-degree polynomial differential equation," Advances in Mathematical Physics, vol. 2020, Article ID 7034591, 7 pages, 2020.

[16] Y. Xu and A. C. J. Luo, "Frequency-amplitude characteristics of periodic motions in a periodically forced van der Pol oscillator," The European Physical Journal Special Topics, vol. 228, no. 9, pp. 1839-1854, 2019.

[17] Y. A. Kuznetsov, Elements of Applied Bifurcation Theory, Springer-Verlag, 2nd edition, 1998.

[18] R. I. Leine and H. Nijmeijer, Dynamics and Bifurcations of Non-Smooth Mechanical Systems, Springer, New York, 2004. 